\title{
Fatores de risco para anemia por deficiência de ferro em crianças e adolescentes parasitados por helmintos intestinais
}

\author{
Luciara L. Brito, ${ }^{1}$ Maurício L. Barreto, ${ }^{1}$ Rita de Cássia R. Silva, ${ }^{2}$ \\ Ana Marlúcia O. Assis, ${ }^{2}$ Mitermayer G. Reis, ${ }^{3}$ Isabel Parraga ${ }^{4}$ \\ e Ronald E. Blanton ${ }^{5}$
}

RESUMO Objetivo. Investigar os fatores de risco para anemia por deficiência de ferro em crianças e adolescentes ( 7 a 17 anos) infectados por helmintos.

Métodos. Foi realizado um estudo transversal com 1709 crianças e adolescentes residentes na cidade de Jequié, Estado da Bahia, Brasil, que apresentavam infecção leve ou moderada por Schistosoma mansoni, Ascaris lumbricoides, Trichuris trichiura e ancilostomídeos. Foram obtidos dados sobre níveis de hemoglobina (hemoglobinômetro portátil), consumo alimentar (inquérito recordatório de 24 horas), infecção parasitária (método Kato-Katz), condições ambientais e domiciliares, renda e escolaridade dos responsáveis. Os fatores de risco para anemia na população foram estudados com base em um modelo hierárquico de causalidade.

Resultados. A prevalência de infecção por T. trichiura, A. lumbricoides, S. mansoni $e$ ancilostomídeos foi de 74,8,63,0, 55,5 e 15,7\%, respectivamente. Constatou-se que 32,2\% das crianças e adolescentes eram anêmicos. Depois do ajuste para variáveis de confusão, os resultados da análise multivariada mostraram que a renda familiar per capita abaixo de um quarto do salário mínimo (27 dólares), o sexo masculino, a faixa etária de 7 a 9 anos e a ingestão inadequada de ferro biodisponível foram significativamente associados à anemia.

Conclusões. As ações para controle da anemia no grupo de maior risco, conforme identificado no presente estudo, devem visar o aumento do consumo de alimentos ricos em ferro e da biodisponibilidade do ferro ingerido, bem como a melhoria das condições sócio-ambientais.

Palavras-chave Consumo de alimentos, anemia, criança, adolescente.

1 Universidade Federal da Bahia (UFBa), Instituto de Saúde Coletiva. Correspondência e pedidos de separatas devem ser enviados a Luciara Leite Brito no seguinte endereço: UFBa, Instituto de Saúde Coletiva, Rua Padre Feijó $28 / 4^{\circ}$ andar, Canela, CEP 40110-170, Salvador, BA, Brasil. Fone: +55-71245-0544; e-mail: luciara@ufba.br

2 UFBa, Escola de Nutrição.

3 Fundação Oswaldo Cruz (FIOCRUZ), Rio de Janeiro (RJ), Brasil.

4 Case Western Reserve University, Departamento de Nutrição, Cleveland $(\mathrm{OH})$, Estados Unidos.

5 Case Western Reserve University, Divisão de Medicina Geográfica.
A anemia por deficiência de ferro constitui-se em um dos problemas nutricionais de maior magnitude no mundo, tendo atingido cerca de 2 bilhões de indivíduos no ano de 1999 número que corresponde a um terço da população mundial (1). A anemia traz inúmeras conseqüências para o organismo de indivíduos de ambos os sexos e de todas as idades. Nos adultos, o principal impacto da anemia é a diminuição da capacidade produtiva (2). Na infância e no período escolar, fases da vida onde existe um aumento das necessidades nutricionais, a anemia tem efeitos negativos sobre o crescimento ponderal e estatural (3) e sobre a aprendizagem escolar (4).

A anemia por deficiência de ferro resulta da combinação de múltiplos fatores etiológicos. Entre as causas imediatas dessa carência destacam-se a 
baixa ingestão de alimentos fontes de ferro, a baixa absorção do ferro ingerido e as perdas desse micronutriente devido a infecções parasitárias. Por exemplo, as infecções helmínticas, cuja prevalência é elevada nos países em desenvolvimento, especialmente entre crianças e adolescentes, podem comprometer os níveis nutricionais de ferro (5). No Brasil, a grande maioria dos estudos sobre anemia se refere a pré-escolares e gestantes. Há uma grande lacuna particularmente no que se refere à prevalência da anemia em escolares e aos fatores de risco associados a essa deficiência nutricional nesse grupo etário.

Tendo em vista tais considerações, realizou-se o presente estudo com o objetivo de investigar os fatores de risco para anemia em uma população de crianças e adolescentes com infecção leve e moderada por $S$. mansoni, $A$. lumbricoides, $T$. trichiura e ancilostomídeos.

\section{MATERIAIS E MÉTODOS}

Trata-se de um estudo de corte transversal, realizado na área urbana do Município de Jequié, localizado na região sudoeste do Estado da Bahia, a $380 \mathrm{~km}$ da capital do Estado, Salvador. Jequié está classificado na $27^{\mathrm{a}}$ posição dentre os municípios do Estado da Bahia segundo o índice geral de desenvolvimento socioeconômico. A economia de Jequié é baseada no cultivo do cacau e da banana e na criação de bovinos e suínos (6). Segundo dados da Secretaria Estadual de Saúde do Estado da Bahia (SESAB), as doenças infecciosas e parasitárias representam a segunda causa de óbito no Município (7).

Jequié possui diversas coleções hídricas, entre elas o Rio de Contas. $\mathrm{Na}$ atualidade, esse rio transformou-se em local de escoamento, sem tratamento prévio, de dejetos sanitários de empresas, hotéis e residências. Devido ao alto índice de poluição, a prevalência de esquistossomose é alta em toda a bacia fluvial do Rio de Contas.

Os participantes do estudo tinham idade entre 7 e 17 anos, eram residentes na área periférica da cidade e foram selecionados nas escolas públicas e através de visita domiciliar. A decisão de buscar os participantes tanto na escola quanto no domicílio baseou-se na constatação, a partir das listagens com nomes dos alunos fornecidas pelas escolas, do alto grau de evasão escolar. Assim, adotou-se a busca ativa nos domicílios, com o intuito de evitar vieses de seleção.

Foram identificados 13771 escolares elegíveis para o estudo, os quais participaram do diagnóstico parasitológico. Desses, 1709 eram portadores de infecção parasitária de intensidade leve ou moderada, a saber: Schistosoma mansoni $<400$ ovos/grama de fezes; ancilostomídeos $<2000$ ovos/grama de fezes; Ascaris lumbricoides < 50000 ovos/grama de fezes e Trichuris trichiura $<20000$ ovos/gramas de fezes. Essas 1709 crianças constituíram a amostra deste estudo.

Para cada indivíduo, foram coletadas duas amostras de fezes em momentos diferentes. Foram feitas duas lâminas de cada amostra, totalizando quatro lâminas por indivíduo. A identificação dos parasitos foi realizada através da técnica quantitativa de Kato-Katz (8). A estimativa do número de ovos foi feita multiplicando-se a constante 24 pelo número de ovos eliminados por grama de fezes em cada lâmina de Kato. As lâminas foram examinadas 2 horas após preparo para permitir a leitura dos ovos de ancilostomídeos.

Foram obtidos ainda dados sobre saneamento ambiental (abastecimento de água, esgoto e coleta pública de lixo), condições de moradia (tipo de construção, número de pessoas por cômodo, instalação sanitária), renda e escolaridade dos responsáveis. Para coleta desses dados utilizou-se um questionário padronizado, aplicado aos chefes de família ou aos responsáveis pelos escolares. $\mathrm{Na}$ construção do indicador das condições ambientais, todas as variáveis foram dicotomizadas e codificadas com o número 1 quando as condições eram insatisfatórias e com o número 0 quando as condições eram satisfatórias. A partir de análises univariadas, foram selecionadas para compor o modelo de regressão logística as variáveis ambientais e domiciliares significativamente associadas a anemia ou a infecções por
S. mansoni, T. trichiura, A. lumbricoides e ancilostomídeos. O sexo, a idade e a escolaridade do chefe de família foram incluídos nos modelos como variáveis de ajuste. Fizeram parte do indicador as variáveis que permaneceram significativamente associadas a anemia ou infecções parasitárias: origem da água, freqüência de abastecimento de água, lugar onde é guardada a água, cuidado com água de beber, existência de vaso sanitário, destino dos dejetos, freqüência de coleta de lixo, existência de terreno baldio com lixo, existência de esgoto a céu aberto, tipo de piso, tipo de domicílio, tipo de pavimentação e tipo de parede. A metodologia adotada na construção desse indicador baseou-se em um estudo realizado por Reichenheim e Harpham (9). O indicador foi totalizado e classificado em estrato adequado (escore amambiental de 0 a 6) e estrato inadequado (escore de 7 a 13).

O consumo alimentar foi determinado pelo método de inquérito recordatório de 24 horas (10). As informações foram fornecidas pela criança. Quando a criança não era capaz de fornecer adequadamente essas informações, solicitava-se a ajuda do responsável pela aquisição, preparação e distribuição dos alimentos. Os inquéritos foram aplicados por nutricionistas dedevidamente treinadas no período de terça-feira a sábado, para evitar o registro de consumo do final de semana, que, normalmente, não reflete a dieta diária (11). Um álbum, contendo os desenhos e porções diferenciadas dos alimentos, foi utilizado para facilitar o processo recordatório do informante, assegurando, dessa maneira, a memelhoria da qualidade da informação (12). As informações referentes ao consumo e à dosagem de hemoglobina (HB) foram coletadas todas em um mesmo dia, após serem obtidos os resultados do exame de fezes. As informações sobre o consumo alimentar foram processadas $u$ tilizando-se o Programa Virtual Nutri (13), desenvolvido pelo Departamento de Nutrição da Escola de Saúde Pública da Universidade de São Paulo (USP). Para o cálculo centesimal da composição das dietas 
foi também utilizada a tabela de composição de alimentos do Estudo Nacional de Despesa Familiar (ENDEF) (14) para alimentos regionais.

Para a avaliação da adequação das dietas em relação à energia foram consideradas as recomendações da Organização das Nações Unidas para a Agricultura e a Alimentação (Food and Agriculture Organization, FAO) e da Organização Mundial da Saúde (OMS) $(15,16)$. O ferro biodisponível foi quantificado através da equação desenvolvida por Monsen e Balintfy (17). Para essa quantificação levou-se em consideração a composição de cada refeição com relação a ferro total, ferro heme, ferro não-heme e quantidade de ácido ascórbico e de carnes. $\mathrm{Na}$ avaliação do consumo alimentar foram levadas em conta as adequações dos consumos de energia e de ferro biodisponível. $\mathrm{O}$ consumo desses nutrientes foi expresso conforme sua adequação percentual às necessidades diárias médias, as quais levam em conta o sexo e a idade da criança. Em seguida, o consumo de nutrientes foi categorizado em três grupos: $<50 \%$; de 50 a $99,99 \%$; e $\geq 100 \%$.

Os níveis de $\mathrm{HB}$ foram medidos utilizando-se um hemoglobinômetro portátil (HEMOCUE, Inc., Laguna Hills, CA, EUA). A coleta de sangue foi realizada através de punção digital com estiletes descartáveis. Em algumas análises os níveis de HB foram utilizados como variável contínua e, em outras, como variável dicotômica (anêmico/não anêmico), sendo considerados anêmicos aqueles indivíduos com concentrações de HB inferiores aos pontos de corte recomendados pela OMS (18) para a definição da anemia: < 11,5 g/dL para crianças de 7 a 11 anos; < 12,0 g/dL para crianças de 12 a 14 anos; < 13 $\mathrm{g} / \mathrm{dL}$ para homens maiores de 15 anos; e < $12 \mathrm{~g} / \mathrm{dL}$ para mulheres maiores de 15 anos. Um quadro severo de anemia foi diagnosticado sempre que a concentração de HB foi inferior a 9,5 g/dL.

Para a comparação das médias de HB segundo variáveis demográficas e sócio-ambientais empregou-se a análise de covariância (ANACOVA). Para a comparação das prevalências de anemia segundo as características
FIGURA 1. Modelo hierárquico do processo de determinação de fatores de risco para anemia ferropriva em indivíduos de 7 a 17 anos

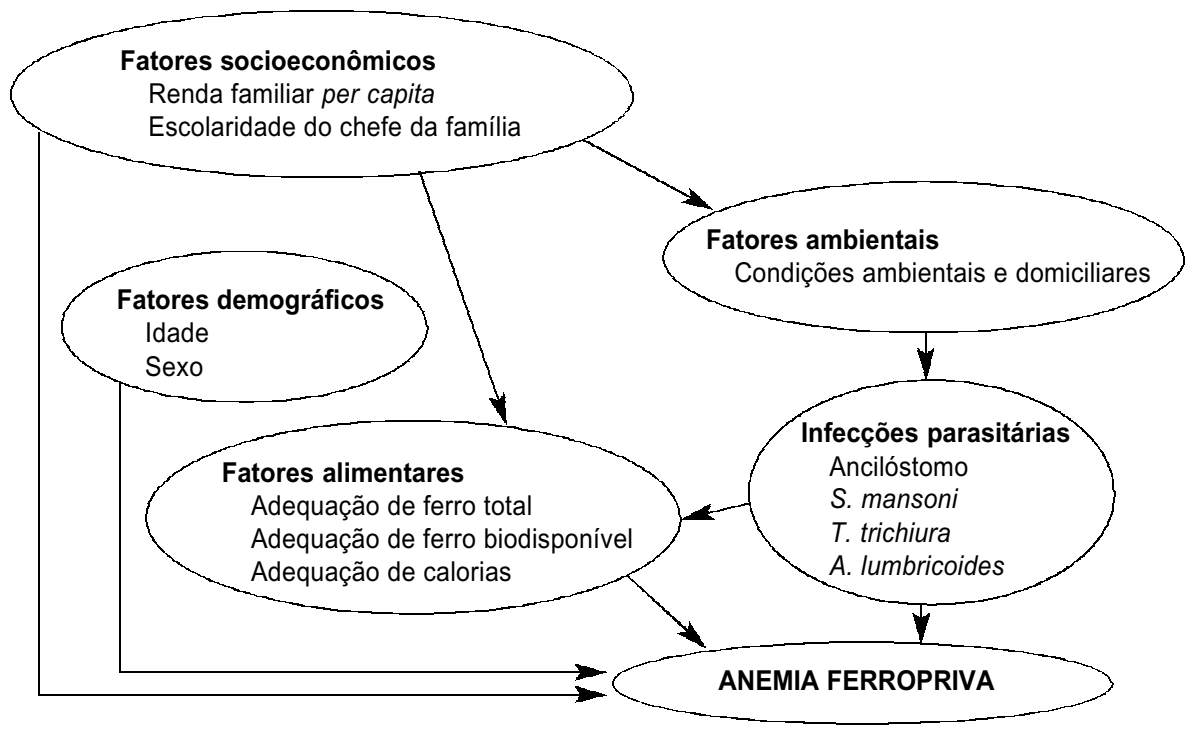

da população estudada empregou-se o teste do qui-quadrado $\left(\chi^{2}\right)$ e $\chi^{2}$ de tendência. Definiu-se um nível de significância de 0,05 para a análise estatística de interesse. Análises bivariadas foram realizadas com o intuito de identificar o conjunto de variáveis que mais contribuíram para a explicação da anemia. A magnitude da associação entre anemia e seus possíveis determinantes foi estimada pelo cálculo da razão de chances (odds ratio, OR), adotando-se o intervalo de confiança a 95\% (IC95\%) como medida de precisão.

Posteriormente, foram realizadas análises multivariadas utilizando-se a regressão logística, a partir de um modelo teórico definido a priori, discriminando os fatores de risco em blocos hierarquizados (figura 1) e respeitando a hierarquia existente entre os níveis de determinação da anemia. A estratégia utilizada para a entrada dos blocos de variáveis foi do tipo forward (processo anterógrado), através do módulo em passos primeiro bloco: variáveis socioeconômicas; segundo bloco: variáveis ambientais; terceiro bloco: variáveis demográficas; e quarto bloco: variáveis de consumo alimentar. Permaneceram no modelo as variáveis que mostraram níveis de significância estatística, segundo um $P<0,10$. Os pacotes estatísticos utilizados foram o Epi Info (versão 6.0) (19) e o Stata (versão 7.0) (20).

O protocolo do estudo foi submetido e aprovado pela comissão de ética do Centro de Pesquisa Gonçalo Muniz da Fundação Oswaldo Cruz (FIOCRUZ). Após o fornecimento de informações detalhadas sobre o projeto e explicação acerca dos procedimentos a que seriam submetidos os participantes, os pais ou responsáveis pela criança ou adolescente assinaram um termo de consentimento informado.

\section{RESULTADOS}

Foram incluídos no estudo 1709 crianças e adolescentes de ambos os sexos com idade entre 7 e 17 anos. A prevalência de infecção por $A$. lumbricoides, S. mansoni, T. trichiura e ancilostomídeos foi de 63,0, 55,5, $74,8 \%$ e $15,7 \%$, respectivamente. A tabela 1 mostra os resultados da análise de variáveis demográficas, socioeconômicas e ambientais. A partir dos dados apresentados na tabela 2, constatou-se que o consumo de ferro biodisponível e a ingestão de calorias eram inadequados (< 100\% dos valores recomendados) na maioria dos participantes. 
TABELA 1. Prevalência de anemia e níveis médios de hemoglobina em indivíduos de 7 a 17 anos segundo variáveis demográficas, socioeconômicas e ambientais, Jequié (BA), Brasil, 1997

\begin{tabular}{|c|c|c|c|c|c|c|}
\hline \multirow[b]{2}{*}{ Variáveis } & \multirow[b]{2}{*}{ No. } & \multirow[b]{2}{*}{$(\%)$} & \multicolumn{2}{|c|}{ Hemoglobina $^{a}$} & \multicolumn{2}{|c|}{ Prevalência de anemia } \\
\hline & & & $\begin{array}{l}\text { Média } \\
\text { (g/dL) }\end{array}$ & $\begin{array}{l}\text { Desvio- } \\
\text { padrão }\end{array}$ & $\%$ & IC95\% \\
\hline \multicolumn{7}{|l|}{ Idade } \\
\hline 7 a 9 anos & 740 & $(29,9)$ & 11,9 & 1,2 & 36,8 & 32,6 a 40,9 \\
\hline 10 a 14 anos & 746 & $(57,0)$ & 12,4 & 1,2 & 30,5 & 27,7 a 33,5 \\
\hline 15 a 17 anos & 223 & $(13,1)$ & 13,2 & 1,5 & 28,7 & 22,7 a 34,7 \\
\hline Masculino & 921 & $(53,9)$ & 12,4 & 1,3 & 34,6 & 31,6 a 37,7 \\
\hline Feminino & 788 & $(46,1)$ & 12,3 & 1,2 & 29,3 & 26,1 a 32,5 \\
\hline & & & \multicolumn{2}{|c|}{$P=0,14^{\mathrm{d}}$} & \multicolumn{2}{|c|}{$P=0,01^{\mathrm{e}}$} \\
\hline \multicolumn{7}{|l|}{ Renda familiar per capita (US\$) ${ }^{c}$} \\
\hline$<27,03$ & 903 & $(53,9)$ & 12,2 & 1,2 & 35,6 & 32,4 a 38,7 \\
\hline 27,03 a 54,06 & 478 & $(28,5)$ & 12,4 & 1,3 & 30,5 & 26,4 a 34,7 \\
\hline Analfabeto & 730 & $(42,9)$ & 12,3 & 1,3 & 35,1 & 31,6 a 38,5 \\
\hline \multicolumn{7}{|l|}{ Primário completo } \\
\hline (4 anos de estudo) & 761 & $(44,7)$ & 12,3 & 1,3 & 31,4 & 28,1 a 34,7 \\
\hline \multicolumn{7}{|l|}{$1^{\circ}, 2^{\circ}$ e $3^{\circ}$ graus } \\
\hline (5 ou mais anos de estudo) & 211 & $(12,4)$ & 12,4 & 1,3 & 25,6 & 19,7 a 31,5 \\
\hline & & & \multicolumn{2}{|c|}{$P<0,23^{a}$} & \multicolumn{2}{|c|}{$P<0,01^{b}$} \\
\hline \multicolumn{7}{|c|}{ Condições ambientais e domiciliares ${ }^{c, f}$} \\
\hline Inadequadas & 705 & $(43,6)$ & 12,4 & 1,3 & 35,3 & 31,8 a 38,9 \\
\hline \multirow[t]{2}{*}{ Adequadas } & 911 & $(56,4)$ & 12,2 & 1,3 & 28,8 & 25,8 a 31,7 \\
\hline & & & \multicolumn{2}{|c|}{$P=0,03^{d}$} & \multicolumn{2}{|c|}{$P=0,01^{\mathrm{e}}$} \\
\hline
\end{tabular}

a Teste de linearidade.

b Teste $\chi^{2}$ de tendência.

${ }^{c}$ Média de hemoglobina ajustada por idade.

d ANOVA.

e Teste do $\chi^{2}$

${ }^{\mathrm{f}}$ Condições adequadas: escore ambiental de 0 a 6; condições inadequadas: escore ambiental de 7 a 13.

TABELA 2. Prevalência de anemia e níveis médios de hemoglobina em indivíduos de 7 a 17 anos segundo ingestão de calorias e ferro biodisponível na dieta, Jequié (BA), Brasil, 1997

\begin{tabular}{|c|c|c|c|c|c|c|}
\hline \multirow[b]{2}{*}{ Ingestão (\% do consumo recomendado) } & \multirow[b]{2}{*}{ No. } & \multirow[b]{2}{*}{$(\%)$} & \multicolumn{2}{|c|}{ Hemoglobina $^{a}$} & \multicolumn{2}{|c|}{ Prevalência de anemia } \\
\hline & & & $\begin{array}{l}\text { Média } \\
(\mathrm{g} / \mathrm{dL})\end{array}$ & $\begin{array}{l}\text { Desvio- } \\
\text { padrão }\end{array}$ & $\%$ & IC95\% \\
\hline \multicolumn{7}{|l|}{ Calorias } \\
\hline$<50,0$ & 578 & $(33,8)$ & 12,2 & 1,3 & 36,9 & 32,9 a 40,8 \\
\hline 50,0 a 99,9 & 979 & $(57,3)$ & 12,4 & 1,3 & 30,5 & 27,7 a 33,4 \\
\hline$\geq 100,0$ & 152 & $(8,9)$ & 12,6 & 1,3 & 25,0 & 18,0 a 31,9 \\
\hline & & & \multicolumn{2}{|c|}{$P<0,01^{b}$} & \multicolumn{2}{|c|}{$P<0,01^{c}$} \\
\hline$<50,0$ & 308 & $(18,2)$ & 12,1 & 1,2 & 36,0 & 30,6 a 41,4 \\
\hline 50,0 a 99,9 & 642 & $(37,9)$ & 12,3 & 1,3 & 35,0 & 29,1 a 40,8 \\
\hline$>100,0$ & 745 & $(43,9)$ & 12,5 & 1,3 & 29,3 & 26,0 a 32,5 \\
\hline & & & \multicolumn{2}{|c|}{$P<0,01^{b}$} & \multicolumn{2}{|c|}{$P=0,02^{\mathrm{c}}$} \\
\hline
\end{tabular}

a Média de hemoglobina ajustada por idade.
b Teste de linearidade.

c Teste $\chi^{2}$ de tendência. 
TABELA 3. Associação entre a anemia e os fatores sócio-ambientais e demográficos em indivíduos de 7 a 17 anos, Jequié (BA), Brasil, 1997

\begin{tabular}{|c|c|c|c|}
\hline Variáveis & OR bruto (IC95\%) & OR ajustado (IC95\%) & $P$ \\
\hline \multicolumn{4}{|l|}{ Renda familiar per capita (US\$) } \\
\hline$<27,03$ & $1,7(1,3$ a 2,3$)$ & $1,5(1,1$ a 2,1$)$ & $<0,01$ \\
\hline 27,03 a 54,06 & $1,4(1,0$ a 1,9$)$ & $1,2(0,9$ a 1,8$)$ & 0,12 \\
\hline$>54,06$ & 1,0 & $1,0^{\mathrm{a}}$ & \\
\hline \multicolumn{4}{|l|}{ Escolaridade do chefe da família } \\
\hline Analfabeto & $1,6(1,1$ a 2,2$)$ & $1,3(0,9$ a 1,9$)$ & 0,05 \\
\hline Primário completo (4 anos de estudo) & $1,3(0,9$ a 1,9$)$ & $1,2(0,9$ a 1,8$)$ & 0,14 \\
\hline $1^{\circ}, 2^{\circ}$ e $3^{\circ}$ graus ( 5 ou mais anos de estudo) & 1,0 & $1,0^{b}$ & \\
\hline \multicolumn{4}{|l|}{ Condições ambientais e domiciliares ${ }^{c}$} \\
\hline Inadequadas & $1,4(1,1$ a 1,7$)$ & $1,2(1,0$ a 1,5$)$ & 0,06 \\
\hline Adequadas & 1,0 & $1,0^{\mathrm{d}}$ & \\
\hline \multicolumn{4}{|l|}{ Idade } \\
\hline 7 a 9 anos & $1,4(1,0$ a 2,0$)$ & $1,5(1,1$ a 2,2$)$ & 0,03 \\
\hline 10 a 14 anos & $1,1(0,8$ a 1,5$)$ & $1,2(0,8$ a 1,6$)$ & 0,40 \\
\hline 15 a 17 anos & 1,0 & $1,0^{\mathrm{e}}$ & \\
\hline \multicolumn{4}{|l|}{ Sexo } \\
\hline Masculino & $1,3(1,0$ a 1,6$)$ & $1,4(1,1$ a 1,7$)$ & $<0,01$ \\
\hline Feminino & 1,0 & $1,0^{f}$ & \\
\hline
\end{tabular}

\section{Prevalência de anemia}

$\mathrm{O}$ valor médio de $\mathrm{HB}$ na população em estudo foi de $12,3 \pm 1,3 \mathrm{~g} / \mathrm{dL}$, com concentrações mínima e máxima de 6,5 e $16,5 \mathrm{~g} / \mathrm{dL}$, respectivamente. A prevalência global de anemia foi de $32,2 \%$, sendo $0,7 \%$ na forma grave $(\mathrm{HB}<9,5 \mathrm{~g} / \mathrm{dL}$ ) e $31,5 \%$ na moderada. Conforme mostra a tabela 1, observouse elevação nos níveis médios de HB ( $P$ $<0,01)$ e redução na prevalência da anemia com aumento da idade $(P<$ $0,05)$. Constatou-se que a prevalência da anemia foi maior nos indivíduos do sexo masculino do que nos indivíduos do sexo feminino $(P<0,01)$. Além disso, a prevalência da anemia caiu com o aumento da renda familiar per capita $(P$ $<0,01)$ e da escolaridade do chefe de família $(P<0,01)$. Houve maior prevalência da anemia em indivíduos cujas condições ambientais e dodomiciliares eram inadequadas (tabela 1). Observou-se que a prevalência da anemia caiu significativamente com o aumento da adequação do consumo de calorias $(P<0,01)$ e de ferro biodisponível $(P<0,05)$ (tabela 2$)$.

\section{Condições sócio-ambientais, fatores demográficos e anemia}

A partir da primeira etapa da análise de regressão logística, identificou-se que a renda familiar per capita e a escolaridade do chefe de família atendiam os critérios previamente estabelecidos para permanecerem no modelo (tabela 3). Houve associação significativa entre renda familiar per capita inferior a um quarto do salário mínimo ( $<27$ dólares) e anemia, mesmo após ajuste pela escolaridade do chefe de família (OR = 1,5; IC95\%: 1,1 a 2,1). Por outro lado, a escolaridade do chefe de família não se associou à anemia de forma significativa após ajuste pela renda familiar per capita. Na segunda etapa da análise, o indicador das condições ambientais e domiciliares foi ajustado para as variáveis selecionadas na etapa anterior; observou-se que a anemia se associou com condições ambientais e domiciliares inadequadas, embora a significância estatística tenha sido limítrofe (OR = 1,2; IC95\%: 1,0 a $1,5)$.

As variáveis demográficas foram ajustadas pelas variáveis socioeconômicas e ambientais selecionadas nas etapas anteriores. $\mathrm{O}$ sexo masculino esteve associado à anemia, confirmando os achados da análise bivariada. Com relação à idade, após ajuste pelas variáveis citadas acima, verificou-se que crianças com idade de 7 a 9 anos apresentaram uma chance 1,5 vez maior de desenvolver anemia do que aquelas na faixa etária de 15 a 17 anos (tabela 3).

\section{Consumo alimentar e anemia}

De acordo com o modelo teórico, a adequação do consumo de ferro biodisponível foi ajustada para as variáveis socioeconômicas, ambientais e demográficas, bem como para as infecções parasitárias (A. lumbricoides, T. trichiura, ancilostomídeos e $S$. 
TABELA 4. Associação entre a anemia e a ingestão de calorias e ferro biodisponível em indivíduos de 7 a 17 anos, Jequié (BA), Brasil, 1997

\begin{tabular}{|c|c|c|c|}
\hline Ingestão (\% do consumo recomendado) & OR bruto (IC95\%) & OR ajustado (IC95\%) & $P$ \\
\hline \multicolumn{4}{|l|}{ Calorias } \\
\hline$<50,0$ & $1,5(1,1$ a 2,0$)$ & $1,2(0,8$ a 1,9$)$ & 0,30 \\
\hline 50,0 a 99,9 & $1,2(0,9$ a 1,6$)$ & $1,1(0,7$ a 1,6$)$ & 0,70 \\
\hline$\geq 100,0$ & 1,0 & $1,0^{\mathrm{a}}$ & \\
\hline \multicolumn{4}{|l|}{ Ferro biodisponível } \\
\hline$<50,0$ & $1,2(1,0$ a 1,5$)$ & $1,6(1,1$ a 2,2$)$ & $<0,01$ \\
\hline 50,0 a 99,9 & $1,2(1,0$ a 1,4$)$ & $1,5(1,0$ a 1,7$)$ & 0,03 \\
\hline$\geq 100,0$ & 1,0 & $1,0^{b}$ & \\
\hline
\end{tabular}

mansoni) e para a adequação do consumo de calorias. Verificou-se que os escolares e adolescentes que consumiam uma dieta com menos de $50 \%$ do valor recomendado de ferro biodisponível apresentaram uma chance 1,6 vez maior de desenvolver anemia do que aqueles que consumiram uma dieta com valor igual ou superior a $100 \%$ do consumo recomendado de ferro (tabela 4 ). Em relação à categoria de consumo entre 50,0 e $99,9 \%$ dos valores recomendados, observou-se que, mesmo após ajuste pelos fatores citados acima, a associação com a anemia permaneceu no limiar de significância estatística. Não se observou associação estatisticamente significativa entre anemia e consumo de calorias após ajuste para as variáveis selecionadas nas etapas anteriores da regressão logística e após ajuste pelas variáveis consumo de ferro biodisponível e infecções parasitárias (tabela 4).

\section{Avaliação do ajuste da modelagem}

Os dados apresentados na tabela 5 mostraram que o modelo composto pelas variáveis socioeconômicas permitiu identificar 9\% dos casos de anemia. Observou-se que esse percentual se manteve em $9 \%$ após a inclusão do indicador das condições ambientais e elevou-se para 16\% quando foi incluída a idade. Quando todas as variáveis significativas foram incluídas na modelagem, uma proporção maior de casos foi identificada (30\%). A inclusão dos blocos de variáveis socioeconômicas, ambientais, reprodutivas maternas e nutricionais contribuiu signicativamente para a modelagem.

$\mathrm{Na}$ tabela 6 estão listados os alimentos mais freqüentes nas principais refeições realizadas pelos participantes. De acordo com os dados, as refeições que mais contribuíram para o fornecimento de ferro foram o almoço e o jantar. Ao analisar a contribuição dos alimentos que são fontes de ferro ou favorecem sua absorção, por refeição, verificou-se uma maior proporção da carne de boi, seguida pelo frango, com quantidade média ingerida menor do que 40 g no caso da carne de boi e de $45 \mathrm{~g}$ no caso do frango. O feijão contribuiu em média com 2,6 e 2,4 mg de ferro no almoço e no jantar, respectivamente. Em relação aos vegetais ricos em vitamina $C$, destacou-se o consumo de laranja, banana, mamão e maracujá.

TABELA 5. Avaliação da contribuição de cada bloco de variáveis para o ajuste do modelo, estudo sobre fatores de risco para anemia por deficiência de ferro em indivíduos de 7 a 17 anos parasitados por helmintos intestinais, Jequié (BA) Brasil, 1997

\begin{tabular}{|c|c|c|c|c|c|}
\hline Bloco de variáveis & $\begin{array}{l}\text { Função } \\
\text { desvio }\end{array}$ & $\begin{array}{l}\text { Graus } \\
\text { de liberdade }\end{array}$ & $\chi^{2}$ & $P$ & $\begin{array}{c}\text { Poder } \\
\text { explicativo (\%) }\end{array}$ \\
\hline Modelo vazio & -1073.6586 & & & & \\
\hline Nível socioeconômico & -1039.3327 & 4 & 18,2 & $<0,01$ & 9 \\
\hline Nível socioeconômico + variáveis ambientais & -978.71463 & 5 & 17,2 & $<0,01$ & 9 \\
\hline $\begin{array}{l}\text { Nível socioeconômico + variáveis ambientais + } \\
\text { variáveis demográficas }\end{array}$ & -971.4818 & 7 & 31,7 & $<0,01$ & 16 \\
\hline $\begin{array}{l}\text { Nível socioeconômico + variáveis ambientais + } \\
\text { variáveis demográficas + consumo alimentar + } \\
\text { infecções parasitárias }\end{array}$ & -950.33673 & 13 & 58,7 & $<0,01$ & 30 \\
\hline
\end{tabular}


TABELA 6. Contribuição dos diferentes alimentos para o fornecimento de ferro de acordo com as refeições realizadas por indivíduos de 7 a 17 anos, Jequié (BA), Brasil, 1997

\begin{tabular}{|c|c|c|c|c|c|}
\hline \multirow[b]{2}{*}{ Refeição } & \multirow[b]{2}{*}{ Alimento } & \multirow{2}{*}{$\begin{array}{l}\text { Porção } \\
\text { média }\end{array}$} & \multicolumn{2}{|c|}{ Quantidade média de ferro } & \multirow{2}{*}{$\begin{array}{l}\% \text { de indivíduos } \\
\text { que consumiram }\end{array}$} \\
\hline & & & $\mathrm{mg}$ & Desvio-padrão & \\
\hline \multicolumn{6}{|l|}{ Desjejum } \\
\hline & Café & $178,3 \mathrm{~mL}$ & 0,7 & 0,6 & 80,4 \\
\hline & Raízes e tubérculos ${ }^{a}$ & $159,9 \mathrm{~g}$ & 0,9 & 0,7 & 7,9 \\
\hline & Frutas $^{\mathrm{b}}$ & $149,3 \mathrm{~g}$ & 1,0 & 1,0 & 2,4 \\
\hline & Pão/biscoito & $77,4 \mathrm{~g}$ & 0,3 & 1,0 & 90,4 \\
\hline & Leite in natura & $139,3 \mathrm{~mL}$ & 0,2 & 0,1 & 15,6 \\
\hline \multicolumn{6}{|l|}{ Almoço } \\
\hline & Fígado & $31,8 \mathrm{~g}$ & 1,5 & 1,4 & 1,2 \\
\hline & Carne de boi & $36,55 \mathrm{~g}$ & 0,9 & 1,8 & 69,8 \\
\hline & Frango & $44,6 \mathrm{~g}$ & 0,6 & 0,6 & 15,8 \\
\hline & Ovo de galinha & $58,1 \mathrm{~g}$ & 1,3 & 1,4 & 4,2 \\
\hline & Feijão & $171,9 \mathrm{~g}$ & 2,6 & 2,4 & 90,2 \\
\hline & Verduras ${ }^{c}$ & $32,9 \mathrm{~g}$ & 0,2 & 0,3 & 22,9 \\
\hline & Farinha de mandioca & $83,3 \mathrm{~g}$ & 0,8 & 0,6 & 39,7 \\
\hline & Frutas $^{b}$ & $95,4 \mathrm{~g}$ & 0,3 & 0,5 & 10,4 \\
\hline \multicolumn{6}{|l|}{ Jantar } \\
\hline & Carne de boi & $36,1 \mathrm{~g}$ & 0,7 & 1,1 & 41,7 \\
\hline & Frango & $22,0 \mathrm{~g}$ & 0,8 & 0,5 & 8,2 \\
\hline & Feijão & $161,2 \mathrm{~g}$ & 2,4 & 2,4 & 42,3 \\
\hline & Frutas $^{b}$ & $134,8 \mathrm{~g}$ & 0,8 & 1,8 & 2,9 \\
\hline & Leite & $148,8 \mathrm{~mL}$ & 0,2 & 0,1 & 5,8 \\
\hline & Pão & $83,9 \mathrm{~g}$ & 0,5 & 1,3 & 33,1 \\
\hline
\end{tabular}

\section{DISCUSSÃO}

A prevalência de anemia observada no presente estudo, de 32,2\%, se assemelha àquela observada por Uchimura, em Maringá, Estado do Paraná (31,7\%) (21). Entretanto, é mais elevada do que a prevalência observada em Aracaju (27,6\%) (22), no Estado de Sergipe, e no Município de Tucano (22,3\%) (23), no Estado da Bahia, em grupos da mesma faixa etária. Contudo, prevalências maiores do que a observada em nosso estudo foram relatadas em Matriz da Luz, Estado de Pernambuco (43\%) (24), e em Osasco, Estado de São Paulo (51\%) (25).

O modelo explicativo adotado no presente trabalho buscou discriminar as relações entre as variáveis na determinação da anemia. Assim, postula-se que a ocorrência da anemia esteja associada a causas estruturais ou básicas, representadas pelas condições socioeconômicas dos indivíduos. As causas imediatas, que compreendem o consumo de alimentos e a exposição às infecções parasitárias, estariam condicionadas diretamente pela condição econômica.

Neste estudo não foi possível analisar as infecções helmínticas intestinais enquanto fatores de risco para a anemia, nem por tipo de parasito nem por intensidade de infecção. Isso se deveu ao fato de que todos os participantes estavam infectados por pelo menos um parasito, o que dificultou a definição de um grupo que pudesse ser utilizado como grupo de comparação. Além disso, o perfil da população era muito complexo, em função da combinação dos diversos graus de infecção por diferentes parasitos. Assim, as infecções parasitárias, no presente estudo, foram consideradas apenas como variáveis potencialmente confundidoras da associação entre a anemia e o consumo alimentar de ferro biodisponível, uma vez que as infecções parasitárias são fatores de risco para anemia (efeito) e estão comumente associadas ao consumo alimentar inadequado.

A renda per capita inferior a um quarto do salário mínimo apresentouse associada à anemia nesta população. Esse achado está de acordo com os resultados de outras investigações $(21,26,27)$. É importante ressaltar que mais de $50 \%$ dos indivíduos que participaram deste estudo pertenciam a famílias que relataram ter renda per capita inferior a um quarto do salário mínimo, o que confirma o quadro de adversidade ambiental e social a que estão submetidos. Os fatores econômicos podem influenciar a ocorrência da anemia de diversas maneiras, em especial pelo papel que exercem sobre a possibilidade de aquisição e utilização de bens e serviços essenciais à manutenção do estado de saúde. Apesar de a prevalência da anemia ser significativamente maior nos 
indivíduos de nível socioeconômico mais baixo, os indivíduos do estrato socioeconômico mais privilegiado também apresentam prevalências relativamente altas da anemia. Isso poderia ser atribuído a fatores (por exemplo, fatores culturais que definem os alimentos preferenciais para consumo) que atravessam as condições de classe e atingem os indivíduos independentemente do nível socioeconômico, e que podem prejudicar tanto o consumo dos alimentos ricos em ferro biodisponível quanto o consumo dos alimentos que favorecem a absorção desse mineral.

Nossos resultados mostraram também que as condições ambientais adversas podem influenciar a ocorrência da anemia, possivelmente por favorecer uma maior exposição dos indivíduos aos processos infecciosos e parasitários. Notadamente, os resultados deste estudo destacam a importância das condições de saneamento do ambiente e do domicílio enquanto determinantes da anemia, dado que a associação permaneceu mesmo após ajuste pela renda familiar per capita.

As crianças com idade entre 7 e 9 anos constituíram o grupo mais vulnerável à anemia. É interessante mencionar que essa relação inversa entre prevalência de anemia e idade repete-se em outras pesquisas. Fujimori et al. observaram que a anemia foi mais freqüente nos escolares com idade abaixo dos 12 anos $(27,5 \%)$, quando comparados com indivíduos de 12 a 14 anos $(15,7 \%)$ e de 15 a 17 anos $(8,8 \%)(27)$. Entretanto, são necessários novos estudos que avaliem a distribuição da anemia nas diversas faixas etárias e as possíveis causas para a maior vulnerabilidade de determinados grupos.

A constatação de que a ocorrência da anemia é mais elevada em crianças e adolescentes do sexo masculino pode ser justificada em parte pela maior necessidade de ferro que indivíduos do sexo masculino apresentam, particularmente pela atividade física mais intensa, já que o ferro é um nutriente essencial na transferência e na formação de estoques de oxigênio para uso durante as contrações musculares e exerce papel essencial na produção oxidativa de energia celular. Além disso, as infecções parasitárias intestinais - condição que também provoca aumento na demanda de ferro - foram mais freqüentes no sexo masculino. Embora esses resultados sejam concordantes com os de Ferreira et al. (24), que identificaram maior ocorrência de anemia no sexo masculino, outras investigações não identificaram diferenças estatísticas significativas na ocorrência da anemia entre os sexos $(22,25)$.

Embora as evidências indiquem um papel relevante dos fatores nutricionais na determinação e evolução da anemia, poucos estudos têm abordado essa questão. Neste estudo, a avaliação da biodisponibilidade do ferro nas dietas possibilitou que se fizesse uma estimativa do ferro absorvido e permitiu avaliar de modo mais consistente a inadequação do consumo dietético de ferro biodisponível e a ocorrência da anemia. Os resultados deste estudo levam a supor que o baixo conteúdo de ferro biodisponível na dieta constitui um fator crítico na determinação da anemia em crianças e adolescentes.

Identificou-se que o feijão foi a principal fonte de ferro na alimentação dos indivíduos investigados. A absorção do ferro disponível nesse alimento depende da presença de outros componentes alimentares na dieta, a exemplo das carnes. É importante ressaltar que, embora fosse alta a freqüência do consumo de carnes, a quantidade consumida por refeição era extremamente pequena. A importância das carnes enquanto favorecedoras da absorção de ferro ganha relevância na medida em que a maior parte do ferro dietético disponível nas dietas dos escolares é do tipo não-heme, proveniente dos alimentos de origem vegetal, como cereais, leguminosas, raízes, verduras e frutas. Muitos estudos têm confirmado o efeito favorecedor das carnes na absorção do ferro, desde que Layrisse et al. (28) demonstraram, pela primeira vez, que a adição de carne de vitela a uma refeição composta de feijão preto ou milho duplicou a absorção do ferro não-heme. O efeito favorecedor das carnes de boi, carneiro, galinha, peixe ou porco, além de fígado, na absorção do ferro, parece estar relacionado ao alto nível de cisteína contido nesses tecidos. Além disso, observou-se que um contingente apreciável de indivíduos não consumiu alimentos fontes de vitamina $C$, outro potente favorecedor da absorção do ferro nãoheme. Destaca-se ainda que esse efeito protetor só é registrado quando a vitamina $C$ é consumida conjuntamente com as fontes de ferro.

O presente estudo fornece evidências de que, além de um baixo consumo de alimentos favorecedores da absorção de ferro não-heme, o grupo investigado apresentou um baixo consumo quantitativo de alimentos que contêm o ferro heme, forma em que o micronutriente é prontamente absrovido (20 a $30 \%$, sem influência do tipo, composição ou combinação de alimentos. Esse padrão de consumo pode explicar, em parte, a alta ocorrência da anemia nesse grupo.

A escassez de dados epidemiológicos sobre o papel da alimentação na determinação da anemia em escolares e adolescentes no Brasil dificulta a análise comparativa dos resultados encontrados neste estudo. Em investigação realizada em Osasco, Município de São Paulo, os resultados sinalizam que a baixa prevalência de anemia $(5,3 \%)$ poderia ser creditada à boa qualidade da dieta consumida pelos adolescentes (29). Uchimura, estudando 334 escolares de Maringá, Estado do Paraná, observou que a anemia estava associada à ausência do consumo habitual de feijão, carnes e frutas (21).

A principal limitação desta investigação provém de seu desenho transversal, o que afeta a interpretação dos resultados, na medida em que não é possível identificar se os fatores de risco foram antecedentes à anemia. É importante salientar que a anemia pode exercer um papel importante na redução do apetite; assim, os estudos transversais não podem estabelecer se o consumo inadequado levou ao quadro anêmico ou se a anemia levou a um consumo inadequado. Dessa forma, o papel dos fatores de risco seria melhor investigado por meio de estudos prospectivos, que permitiriam comparar os coeficientes de incidência 
da anemia e os graus de exposição aos fatores de risco, estabelecendo diferenciais de risco.

A técnica utilizada para investigar o consumo alimentar também apresenta algumas limitações. Embora o recordatório de 24 horas seja um método rápido, de custo relativamente baixo, e que pode ser utilizado com indivíduos em todos os níveis de escolaridade, o sucesso dessa estratégia depende da memória e do grau de motivação do entrevistado, como também da persistência e da habilidade dos entrevistadores para obtenção de estimativas acuradas das porções consumidas. Além disso, não retrata o consumo habitual da população estudada, informação que seria fundamental para determinar a contribuição da dieta no desenvolvimento da anemia (12).

Em conclusão, a elevada prevalência da anemia observada no presente estudo mostra a necessidade de se incluir a vigilância do estado nutricional referente ao ferro como parte dos cuidados permanentes com a saúde de indivíduos na faixa etária de 7 a 17 anos, especialmente em populações vivendo em condições semelhantes às descritas aqui. A magnitude do problema mostrou que é necessária a adoção de medidas imediatas para o controle dessa deficiência nutricional. As estratégias

\section{REFERÊNCIAS}

1. Stoltzfus RJ. Defining iron-deficiency anemia in public health terms: a time for reflection. J Nutr 2001;131(2S-2):565S-567S.

2. Haas JD, Brownlie T. Iron deficiency and reduced work capacity: a critical review of the research to determine a causal relationship. J Nutr 2001;131(2S-2):676S-688S

3. Lawless JW, Latham MC, Stephenson LS, Kinoti SN, Pertet AM. Iron supplementation appetite and growth in anemic Kenyan primary school children. J Nutr 1994;124(5):645- 654.

4. Pollitt E. Early iron deficiency anemia and later mental retardation. Am J Clin Nutr 1999; 69(1):4-5.

5. Walter-Smith JA, Mcneish AS. Diarrhoea and malnutrition in childhood. Oxford: Butterworth- Heinemann; 1986.

6. Superintendência de Estudos Econômicos e Sociais da Bahia. O perfil financeiro dos municípios baianos: 1993-1997. Salvador: SEI; 2001.

7. Bahia, Secretaria de Saúde, Centro de Informações de Saúde. Anuário estatístico. Informações de saúde. Dados por município. Salvador: SESAB; 1996

8. Katz N, Chaves A, Pellegrino J. A simple device for quantitative stool thick-smear technique in Schistosoma mansoni. Rev Inst Med Trop Sao Paulo 1972;14(6):397-400.

9. Reichenheim ME, Harpham T. Perfil intracomunitário da deficiência nutricional: estudo de crianças abaixo de 5 anos numa comunidade de baixa renda do Rio de Janeiro (Brasil). Rev Saude Publica 1990;24(1):67-79.

10. Gibson RS. Principles of nutritional assessment. New York: Oxford University Press; 1990. Pp. 37-38.

11. Willet W. Nutritional epidemiology. New York: Oxford University Press; 1990.

12. Magalhães LP, Oliveira VA, Santos JM. Guia para estimar consumo alimentar. Salvador: Universidade Federal da Bahia, Núcleo de Pesquisa de Nutrição e Epidemiologia; 1996.

13. Philippi ST, Szarfarc SC, Laterza CR. Virtual Nutri - versão 1 for Windows. Sistema de
Análise Nutricional. São Paulo: Departamento de Nutrição, Faculdade de Saúde Pública, Universidade de São Paulo; 1996.

14. Fundação Instituto Brasileiro de Geografia e Estatística, Estudo Nacional de Despesa Familiar (ENDEF). Tabela de composição de alimentos. 3a ed. Rio de Janeiro: IBGE; 1985.

15. Energy and protein requirements: Report of a joint $\mathrm{FAO} / \mathrm{WHO} / \mathrm{UNU}$ expert consultation. Geneva: WHO; 1985. (WHO Technical Report Series 724). vitamina B12. Informe de una consulta mixta FAO/OMS de expertos. Roma: OMS; 1991.

17. Monsen ER, Balintfy JF. Calculating dietary iron bioavailability: refinement and computerization. J Am Diet Assoc 1982;80(4):307311.

18. Preventing iron deficiency in women and children: background and consensus on key technical issues and resources for advocacy, planning and implementing national programmes. New York: International Nutrition Foundation and Micronutrient Initiative; 1998. Pp. 1-60.(UNICEF/ UNU/ WHO/MI technical workshop). Disponível em: http:/ /www.inffoundation.org/ publications.html\#1. Acessado em 13 de novembro de 2003.

19. Dean AG, Dean JA, Coulombier D, Brendel KA, Smith DC, Burton AH, et al. Epi Info, Version 6.02: a word processing, database and statistics program for epidemiology on microcomputers. Atlanta, Georgia: Centers for Disease Control and Prevention; 1994.

20. Stata Corporation. Stata statistical software, Release 7.0. College Station: Stata Corporation; 1997.

21. Uchimura TT. Anemia e desnutrição em escolares ingressantes nas escolas estaduais de Maringá-PR [dissertação de mestrado]. São Paulo: Faculdade de Saúde Pública, Universidade de São Paulo; 1994.

22. Tsuyuoka R, Bailey JW, Guimarães AMDN, Gurgel RQ, Cuevas LE. Anemia and intestinal
16. Necesidades de vitamina A, hierro, folato y básicas para combater a anemia devem incluir medidas como sucomo suplementação medicamentosa de ferro, fortificação de alimentos com ferro e educação nutricional. A essas estratégias devem-se somar a prevenção e o controle das parasitoses intestinais (18). Tais medidas devem ser tomadas em conjunto e monitoradas permanentemente. Em locais onde grande parte da população em idade escolar freqüenta a escola, o tratamento da anemia pode ser implantado com certa facilidade, já que as escolas podem desenvolver programas tanto de combate às parasitoses quanto de promoção de uma alimentação saudável. parasitic infections in primary school students in Aracaju, Sergipe, Brazil. Cad Saude Publica 1999;15(2):413-421.

23. Faria AC, Pontes AC, Menezes JF, Cantalice TC, Anjos LMR, Freire AP, et al. Estudo da prevalência de parasitas intestinais associada a anemia em crianças de 2 a 12 anos, residentes em três distritos de do município de Tucano no Estado da Bahia. VI Congresso Brasileiro de Saúde Coletiva. Salvador; 1997. Pp. 525-526.

24. Ferreira MR, Souza W, Pérez EP, Lapa T, Carvalho $A B$, Furtado $A$, et al. Intestinal helminthiasis and anaemia in youngsters from Matriz da Luz, district of São Lourenço da Mata, state of Pernambuco, Brazil. Mem Inst Oswaldo Cruz 1998;93(3):89-93.

25. Stefanini MLR, Colli C, Lerner BR. Anemia e desnutrição em escolares da rede pública do município de Osasco, São Paulo, Brasil. Cad Saude Publica 1995;11(3):439-447.

26. Molina MCB, Leão MAC, Gross R, Schell B, Strock U, Brunken B. Nutritional status of children of urban low income communities. Rev Saude Publica 1989;23(2):89-97.

27. Fujimori E, Szarfarc SC, Oliveira IMV. Prevalência de anemia e deficiência de ferro em adolescentes do sexo feminino: Taboão da Serra, SP, Brasil. Rev Lat Am Enfermagem 1996;4(3):49-63.

28. Layrisse M, Martinez-Torres C, Roche M. Effect of interaction of various foods on iron absorption. Am J Clin Nutr 1968;21(10):11751183.

29. Lerner BR. A alimentação e anemia carencial em adolescentes [dissertação de mestrado]. São Paulo: Faculdade de Saúde Pública, Universidade de São Paulo; 1994.

Manuscrito recebido em 24 de fevereiro de 2003. Aceito em versão revisada em 11 de julho de 2003. 
ABSTRACT Objective. To investigate risk factors for iron-deficiency anemia in children and adolescents (7 to 17 years of age) with intestinal helminthic infections.

Risk factors

for iron-deficiency

anemia in children and

adolescents with intestinal

helminthic infections

Methods. A cross-sectional study was carried out with 1709 children and adolescents living in Jequié, a town in the state of Bahia, Brazil, who had mild to moderate infection by Schistosoma mansoni, Ascaris lumbricoides, Trichuris trichiura, or hookworms. We obtained data concerning hemoglobin levels (using a portable hemoglobinometer), dietary habits (24-hour dietary recall), parasitic infections (KatoKatz method), sanitary conditions (water supply, sewage connection, garbage collection), housing conditions (type of construction, number of persons per room), income, and amount of schooling of the parents or guardians. The risk factors for anemia were studied based on a hierarchical model of causality.

Results. The prevalence of parasitic infection was $74.8 \%$ for T. trichiura, $63.0 \%$ for A. lumbricoides, $55.5 \%$ for S. mansoni, and $15.7 \%$ for hookworms. Among the children and adolescents studied $32.2 \%$ were anemic. After adjustment for confounding variables, the results of the multivariate analysis showed that the following variables were significantly associated with anemia: per capita family income below US\$27 (equal to one fourth of the Brazilian minimum wage), male sex, age of 7 to 9 years, and inadequate intake of bioavailable iron.

Conclusions. The initiatives to control anemia in the group at greatest risk, as identified in this study, should seek to increase the consumption of iron-rich foods, boost the bioavailability of the iron ingested, and improve socioenvironmental conditions. 